Special issue of the International Conference on Computational and Experimental Science and Engineering (ICCESEN 2014)

\title{
Digital Fabrication of Mathematical Models via Low-Cost 3D FDM Desktop Printer
}

\author{
Y. GÜR* \\ Balıkesir University, Mechanical Engineering Department, Balıkesir, Turkey
}

\begin{abstract}
Additive manufacturing technology is developed throughout the 1980's and 1990's. This technology makes the realization of mathematical models much easier. The challenge focused is here to fabricate real objects from very complex mathematical models by using a low-cost 3D fused deposition modeling desktop printer. Thus this brings the intangible mathematical expressions to life. In this study it is illustrated that complex mathematical expressions are not only numbers and symbols but also they are real life objects. In the examples, some mathematical functions such as Möbius strip, Schwartz functions and their boundary conditions are introduced and then digitally fabricated. This work shows that how complex functional mathematical models that cannot be manufactured with classical engineering methods or very difficult to produce can be fabricated by using a 3D FDM desktop printer.
\end{abstract}

DOI: $10.12693 /$ APhysPolA.128.B-100

PACS: 01.50.H-, 02.60.Cb, 07.05.Tp, 81.20.Hy

\section{Introduction}

3D printing technology is developed by Hull throughout the 1980's under the name stereolithography and it is patented in 1986 as "Apparatus for production of threedimensional objects by stereolithography" $[1-6]$. This $3 \mathrm{D}$ printing technology makes the realization of mathematical models much easier than ever. 3D printers allow researchers, designers to quickly fabricate real life objects instead of intangible models of their designs. The models have various uses such as prototypes which can be used for design testing for different area of engineering. This makes time saving, less expenses, faster producing prototype [7].

Production of mathematical models, especially having intricate details, with subtractive manufacturing methodology whose material is removed from a solid block by using milling, lathing, drilling etc. methods can be tricky and very difficult. Using 3D printing technology mitigates the above mentioned problems [8].

The challenge focused is here to fabricate real objects from complex mathematical models such as pseudo torus and Möbius strip by using a low-cost 3D fused deposition modeling (FDM) desktop printer. Thus this brings the abstract mathematical expressions to life.

\section{Fused deposition modeling process}

In 1989, Scott Crump has patented an apparatus for creating three-dimensional objects by fused deposition modeling (FDM) method [9]. In this method, a dispensing extrusion nozzle head moves along $X$, and $Y$ axes while building base is lowered down or tip of the nozzle is higher up in $Z$ axis in order to create a threedimensional object. Building up material is heated up

*e-mail: ygur@balikesir.edu.tr to material's solidification temperature and discharged from the extrusion nozzle onto the building platform at a controlled rate. Thin layer of solidified material is deposited upon the previous layer until the object is fabricated (see Fig. 1).

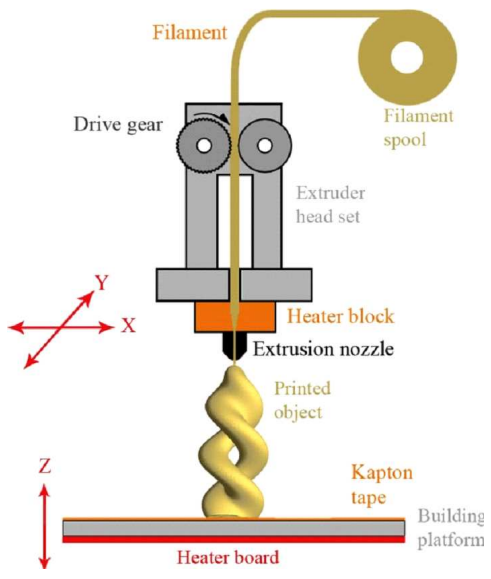

Fig. 1. Fused deposition modeling process [10].

Thermoplastics such as acrylonitrile butadiene styrene (ABS) or polylactic acid (PLA) can be used for building material. If necessary, support structure, which holds the building part in upright position on the building platform, can also be generated during the deposition process.

\section{Pre-processing for $3 \mathrm{D}$ printing of the models}

3D printing process starts with creating of the mathematical models of the objects to fabricate. Mathematical models are generated and visualized by using freeware K3DSurf v. 0.6.2 mathematical modeling program through the evaluation of the below mathematical equations [11] for pseudo torus; 


$$
\begin{aligned}
& \left.F(x, y, z)=\left(\sqrt{\left(\frac{x}{2.7}\right)\left(\frac{x}{2.7}\right)+(y \sin (0.436332 x)+z \cos (0.436332 x))(y \sin (0.436332 x)+z \cos (0.436332 x)}\right)-3\right)^{2} \\
& \quad+(y \cos (0.436332 x)-z \sin (0.436332 x))(y \cos (0.436332 x)-z \sin (0.436332 x))-3=0
\end{aligned}
$$

where $x$ varies from -14 to $8, y$ varies from -5 to 5 , and $z$ varies from -6 to 6 .

$$
x(-14,8) ; y(-5,5) ; z(-6,6)
$$

and parametric equations of the Möbius strip

$$
\begin{aligned}
& X=f(u, v)=\left[20+v \cos \left(\frac{1}{2} u\right)\right] \cos (u)=0, \\
& Y=g(u, v)=\left[20+v \cos \left(\frac{1}{2} u\right)\right] \sin (u)=0, \\
& Z=h(u, v)=v \sin \left(\frac{1}{2} u\right)=0,
\end{aligned}
$$

where $u$ varies from 0 to $4 \pi$ and $v$ varies from 0 to $2 \pi$.
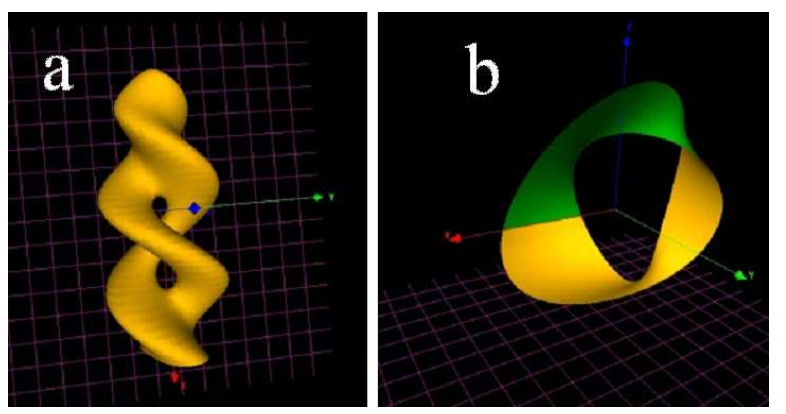

Fig. 2. (a) Mathematical model of pseudo torus,

(b) mathematical model of Möbius strip.

First, $x, y, z$ coordinate values are calculated from the above mentioned Eq. (1) for the pseudo torus and from Eqs. (2)-(4) for the Möbius strip and then visualized by the K3DSurf program in the range of boundary conditions (see Fig. 2a and b). Then, the two models are constructed from these $x, y, z$ coordinate values and later on exported to Wavefront's ".obj" file format. These mathematical models can be thickened by software such as Rhinoceros in order to create solid models. It is necessary to slice the models into sequential layers to print these solid models by a 3D FDM printer. MakerBot ${ }^{\circledR}$ MakerWare $^{\text {TM }}(\mathrm{MW})$ slicing software is used to slice the models (see Fig. 3a and b) [12]. The thicknesses of the layers are $150 \mu \mathrm{m}$. In this slicing step, not only the models sliced but also travel movements of the extrusion nozzle are created and support structures, to hold the solid model in upright position on the building platform, are generated (see Fig. 3b). Printing time and amount of material to use are also calculated and printing preview is allowed. Finally, the data obtained is either directly sent to the 3D printer or converted ".x3g" format and saved on a SD card.

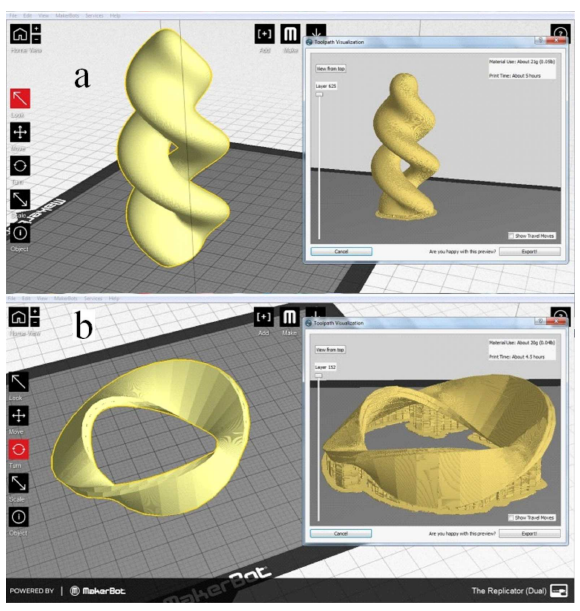

Fig. 3. (a) Slicing the pseudo torus, (b) support structures for Möbius strip.

\section{3D printing of pseudo torus and Möbius strip}

The models were fabricated by using Flashforge Creator dual extruder 3D FDM printer. Building material was ABS plastic and filament having $1.75 \mathrm{~mm}$ in diameter is used. Pseudo torus and Möbius strip are sliced into 625 layers and 152 layers, respectively. Building platform is heated up $110^{\circ} \mathrm{C}$ to provide good bonding for the ABS plastic while heating the extrusion nozzle up to $230^{\circ} \mathrm{C}$. $3 \mathrm{D}$ printing process is taken approximately $5 \mathrm{~h}$ for both parts. After the printings of the models were completed, support structures on the Möbius strip are broken off by hand and both fabricated parts are polished in an acetone vapor bath (see Fig. 4a and b).

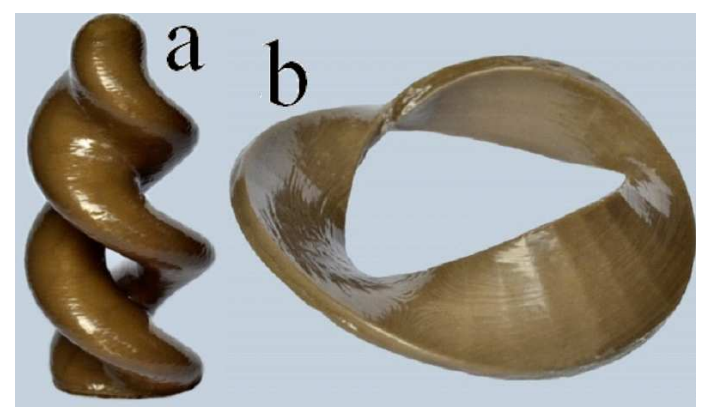

Fig. 4. (a) Fabricated pseudo torus, (b) fabricated Möbius strip. 


\section{Conclusion}

This study highlights the potential of the 3D FDM printing technology on the digital fabrication of mathematical models which is difficult to manufacture or cannot be manufactured with classical engineering methods.

The work presented here shows that intangible complex mathematical expressions can be real life 3D objects by using a low cost 3D FDM printer. Through the pseudo torus and Möbius strip it is also illustrated that those mathematical expressions are not only symbols and numbers but also they are three-dimensional real life objects. These three-dimensional objects allow to illustrate concepts in mathematical field. Tangible physical models are important for hands-on active teaching and learning. Because of this, printed mathematical models can be used for educational purposes and better representations. They can also be used as functional prototypes.

\section{Acknowledgments}

The work described in this paper is supported by the Ballkesir University's Scientific Research Projects Unit through the Project No. Mak. BAP: 2013.0003.

\section{References}

[1] C.W. Hull, U.S. Patent 4575330 (publication date: 3.11.1986).

[2] C.K. Chua, K.F. Leong, C.S. Lim, Rapid Prototyping: Principles and Applications, 2nd ed., World Sci., Singapore 2003, p. 1.
[3] K.G. Cooper, Rapid Prototyping Technology, Selection and Application, Marcel Dekker, New York 2003 (e-book).

[4] S. Crawford, How 3-D Printing Works, http://computer.howstuffworks.com/3-dprinting1.htm, 2013.

[5] I. Gibson, D. Rosen, B. Stucker, Additive Manufacturing Technologies, Rapid Prototyping to Direct Digital Manufacturing, Springer, 2010 (online).

[6] O. Knill, E. Slavkovsky, arXiv:1306.5599 [math.HO], 2013.

[7] V.M. Chapela, M.J. Percino, F.D. Calvo, F. Calvo, L. Trinidad, in: Proc. World Congress on Engineering, WCE 2013, London (UK), Vol. I, 2013.

[8] H. Segerman, The Math. Intelligencer 34, 56 (2012).

[9] S.S. Crump, U.S. Patent 5121329, "Apparatus and method for creating three-dimensional objects" U.S. Class: 364/468, ASSIGNEES: Stratasys, Inc., Minneapolis, MN (field: Oct. 30, 1989 - date of patent: Jun. 9, 1992).

[10] Y. Gür, Mol. Cellul. Biomech. 11, 249 (2014).

[11] A. Taha, K3DSurf Software Package, http://k3dsurf.sourceforge.net/ (30.09.2014).

[12] MakerBot ${ }^{\circledR} \quad$ MakerWare ${ }^{\mathrm{TM}}$ 3-D Printing Software, accessed 30.09.2014, retrieved from http://www.makerbot.com/support/makerware/ documentation/slicer/. 